\title{
Identifying Risk Factors for Self-reported Mental Health Problems in Psychiatry Trainees and Psychiatrists in Mexico
}

\author{
Ana Fresán ${ }^{1}$ (1) - Diana Guízar-Sánchez ${ }^{2}$ (D) - María Yoldi-Negrete ${ }^{1}$ (I) - Rebeca Robles-García ${ }^{1}$ (I) $\cdot$ \\ Carlos-Alfonso Tovilla-Zárate $^{3}$ (D) $\cdot$ Gerhard Heinze $^{2}$ (D) $\cdot$ María-Elena Medina-Mora $^{2}$ (D)
}

Received: 11 February 2021 / Accepted: 28 June 2021 / Published online: 21 July 2021

(C) Academic Psychiatry 2021

\begin{abstract}
Objective The objective was to determine and compare demographic features, professional activities and adversities, physical health conditions, and self-care behaviors related to the most frequently self-reported mental health problems among psychiatrists and psychiatry trainees.

Methods A cross-sectional, retrospective, comparative study was conducted on a total of 330 (48.2\%) psychiatry trainees and $355(51.8 \%)$ psychiatrists from Mexico through an online survey. Demographic features, professional activities and adversities, physical and mental health problems, self-care behaviors, and social support were examined. Comparative analyses and multiple logistic regression models were performed.

Results Major depression, anxiety, and burnout were the most common mental health problems reported with a higher frequency of anxiety disorders in psychiatry trainees. Being a woman, having a physical health problem, and lack of restful sleep were the main risk factors in both groups. Consultation in the government sector and having patients with severe suicidal ideation affected more psychiatry trainees. Perceived discrimination and inadequate eating schedules were risk factors for mental health problems for psychiatrists.

Conclusion Psychiatry trainees constitute a vulnerable group for anxiety disorders. Particular attention should be paid to how students cope with the training experience to determine whether additional support is required. These professionals face major stressors leading to a high prevalence of depression, burnout, and anxiety. Encouraging psychiatrists to have better health habits is a step in the right direction, which must be accompanied by tangible organizational avenues to do so and creating a culture that truly promotes self-care.
\end{abstract}

Keywords Psychiatrists $\cdot$ Psychiatry trainees $\cdot$ Mental health problems $\cdot$ Self-care $\cdot$ Burnout

Physicians are at a greater risk of developing psychiatric morbidities due to the high levels of stress resulting from the significant physical and psychological pressure related to their health care activities, in addition to personal pre-existing vulnerabilities (such as their personal and family history) [1]. Some studies suggest that the competitive culture and

Ana Fresán

a_fresan@yahoo.com.mx; fresan@imp.edu.mx

1 Instituto Nacional de Psiquiatría Ramón de la Fuente Muñiz, Mexico City, Mexico

2 Universidad Nacional Autónoma de México, Mexico City, Mexico

3 Universidad Juárez Autónoma de Tabasco, Comalcalco, Tabasco, Mexico prioritization of others, an intrinsic feature of the medical profession, can lead to the emergence of mental health problems [2]. The field of psychiatry contributes several unique stressors and risk factors to the development of mental health problems, including depression, anxiety, suicide, and burnout $[3,4]$ as psychiatrists care for people with illnesses that are often chronic and incurable [5]. They must cope with patient violence and suicide, stigma, and limited resources as well as increasingly complex administrative and legal frameworks related to their practice. In Mexico, there are six health care and insurance systems, with separate health facilities, pharmacies, and unions. This includes an employer-sponsored system partly subsidized by the government and another five government health care systems that include government workers, workers from the national oil company, and the military. There is also an active private insurance health care system. Unfortunately, 
there is little coordination among these health care systems [6] that may particularly affect psychiatry trainees as in the government sector most of the clinical activities are largely staffed by trainees in addition to their role as students. On the other hand, psychiatrists working in the private sector may neglect their personal care by saturating their time with multiple consultations.

Psychiatrists report a higher impact on their level of distress caused by the doctor-patient relationship than other physicians $[7,8]$. The nature of the psychiatrist-patient relationship is unique among medical specialties, as psychiatrists themselves become "tools" in their profession, which likely allows emotions to intensify or to be affected in the context of their clinical work [9]. Psychiatrists' ability to identify and handle emotions provides greater awareness of and sensitivity to human suffering, while dealing with troubled people continuously over extended periods of time makes them more vulnerable to vicarious trauma and compassion fatigue [10]. Moreover, most psychiatrists experience stressful adversities related to their clinical practice such as attacks by violent patients or hostile relatives of patients, and as a result of managing patients with severe suicidal ideation $[9,11]$ or experiencing patient suicide [12]. They also may face additional strains that increase their risk of mental health problems such as excessive work hours, litigations, a generally solitary professional practice coupled with perceived stigma regarding their profession from the general population and even from their own colleagues [13].

In addition, physicians are known to take poor care of themselves. In an extensive review of physician wellness, Wallace et al. in 2009 described how they tend to work even when sick, expect colleagues to do the same, are unlikely to have a family doctor, often rely on avoidance and denial as coping strategies, self-diagnose and self-prescribe treatments, and in general tend to neglect their own health [1]. Health care organizations are yet another factor leading to poor self-care, by failing to provide resources for basic needs such as rest, physical activity, and nutrition.

As in psychiatrists, mental health problems have been reported as common experiences during residency training [14]. Being a psychiatry trainee confers additional risks that could affect their mental health: long working hours including night shifts, a sense of vulnerability to possible aggression by patients, the imbalance between their professional experience and the responsibility of treating patients, academic demands, and learning-curve errors perceived as failures $[13,15,16]$, together with poor-quality relations within institutions: (1) abuse, harassment, or discrimination by supervisors, peers, and other health care providers, and (2) a collaborative climate and sense of belonging are not fostered [17].

Despite this adverse scenario, psychiatrists generally report being satisfied with their daily practice. In a study by
Cordoba et al. in 2009, comprising 19 Latin American countries, 94\% ( $n=994)$ of the Mexican psychiatrists surveyed reported being satisfied, reflecting a good level of commitment with the profession despite the adversities previously mentioned [18]. Nevertheless, the small number of psychiatrists in Mexico (with an estimated rate of 3.68 per 100,000 inhabitants) and the uneven distribution across the country (60\% of psychiatrists are concentrated in three cities) [19] make them more exposed to factors that can affect their mental health and increase their risk of developing a psychiatric disorder. Indeed, factors such as the workload and long shifts (over $12 \mathrm{~h}$ ), specific to the practice of medicine in Mexico, could further increase the difficulties faced by these specialists [20, 21].

This study provides a retrospective assessment of the main demographic features, professional activities and adversities, physical health problems, and self-care behaviors related to self-reported mental health problems in Mexican psychiatrists and psychiatry trainees. We hypothesize that (1) depression, burnout, and anxiety disorders will be the most prevalent selfreported mental health problems in both psychiatrists and psychiatry trainees; (2) being a woman, having a physical health problem, not having restful sleep, and greater distress will be the most important risk factors for presenting mental health problems in both psychiatry trainees and psychiatrists; and (3) psychiatry trainees will be more affected by their working activities than psychiatrists.

\section{Methods}

This cross-sectional, comparative, retrospective study of Mexican psychiatrists and psychiatry trainees was designed to be used in an online survey. The authors assert that all procedures contributing to this work comply with the ethical standards of the relevant national and institutional committees on human experimentation and with the Helsinki Declaration of 1975, as revised in 2008. The contents of the survey and all study procedures were approved by the Ethics and Research Committees of a psychiatric clinical facility at Mexico City dedicated to research and clinical attention (No. 09-CEI-01020170316).

\section{Participants}

Recruitment was performed using a convenience sample approach with psychiatry trainees and psychiatrists in the country who were willing and able to participate. Psychiatry trainees in Mexico are medical doctors admitted for a 4-year residency program; residents from the first to the fourth year of residency were invited to participate. Psychiatrists are medical doctors who have graduated from the medical specialty in psychiatry currently practicing as specialists. A study 
invitation was circulated, by email and social media (Facebook and WhatsApp), with a link explaining the nature and procedures of the study to participants at the beginning of the online survey, first by stating the anonymity of the survey, then explaining that questions were related to physical and mental health, hobbies, and adversities related to the profession, that the study was approved by the Ethics and Research Committees, and that consent to participate could be withdrawn at any time by dropping out of the survey. A link to an electronic information sheet and consent form was included. Those who agreed to participate and provided electronic consent proceeded to complete the survey. Recruitment was carried out from January 2018 to December 2018.

\section{Assessment Procedure}

The survey was conducted in Spanish and took approximately 25-30 min to answer. It was divided into four sections: demographic features, professional activities and adversities, physical and mental health problems, and self-care behaviors and social support. The first section, "Demographic Features," included questions related to the subjects' age, sex, marital status, and whether they had children. The second section, "Professional Activities and Adversities," included current clinical consultations in both the government and the private sectors, the number of hours per week spent on these activities, and the current maximum working hours per day (excluding the continuous 36-h medical shift schedule). It asked about professional adversities since the beginning of the psychiatry residency and included receiving attacks (physical, verbal, or psychological), the identity of the assailant (patient, patient's relative, or colleague), lawsuits, being the attending physician of patients with suicidal ideation or who committed suicide, and perceived discrimination (discrimination dimension of the Spanish version of King's Internalized Stigma Scale) [22, 23] as a psychiatry trainee or psychiatrist. The third section included self-report questions on "Physical and Mental Health Problems." Participants were asked about the presence of any physical health problem (respiratory, cardiovascular, endocrinological, musculoskeletal, or gastrointestinal diseases), mental health problems (such as major depression, anxiety disorders, burnout, and suicidal ideation) during their professional careers, and whether they had received specialized treatment for these problems. Perceived distress was also assessed on a 10-point visual analogue scale ( 0 - no distress at all; 10-maximum perceived distress). The last section, "Self-care Behaviors," included questions related to eating patterns (number of meals a day, eating schedules, type of diet-hypercaloric, hypocaloric, balanced), exercise (type of exercise - aerobic, anaerobic; frequency; and duration), sleep (number of hours, restful/unrestful sleep), and engagement in social activities outside the workplace.

\section{Statistical Analyses}

Descriptive information was determined by frequencies and percentages for categorical variables and means and standard deviations (S.D.) for continuous variables. The results of the four sections in the survey answered by psychiatry trainees and psychiatrists were compared using chi-square tests $(\chi 2)$ for categorical variables, and independent sample Student's ttests for continuous variables. To determine the effect sizes of the comparative analyses, Cramer's V for chi-square tests and Cohen's $d$ for t-tests were carried out, with the results being interpreted as small $(0.2-0.3)$, medium $(0.4-0.7)$, and large $(>0.8)$.

Demographic features (sex, age, marital status, children), professional activities (clinical consultations in the government and the private sectors, hours spent on these activities, maximum number of working hours per day), and adversities (patients with suicidal ideation, patients who committed suicide, lawsuits, attacks, and perceived discrimination), presence of any physical health problem, perceived distress, and self-care behaviors (meals per day, caloric consumption, eating schedules, exercise, hours of sleep, restful sleep, social activities) were entered as possible predictors of each of the three most frequent self-reported mental health problems (major depression, anxiety disorders, and burnout) in multiple logistic regression analyses with the backward stepwise modeling approach. The Hosmer and Lemeshow test was used to determine goodness of fit. The most calibrated final models were reported, one for psychiatry trainees and another for psychiatrists, which included the variables that remained significant after the backward stepwise process. All analyses were performed using the SPSS version 21 for Windows PC and the alpha value for tests was set at $\mathrm{p}<0.05$.

\section{Results}

\section{Demographic Features}

A total of $330(48.2 \%)$ psychiatry trainees and 355 (51.8\%) psychiatrists from 29 of 32 states in Mexico completed the survey. Considering the last report [19] on the number of psychiatry trainees and psychiatrists in our country, $45.8 \%$ $(n=720)$ of psychiatry trainees and only $7.1 \%(n=4999)$ of psychiatrists answered the survey. As expected, psychiatry trainees were younger (28.4 years old, S.D. $=2.1)$ than psychiatrists (42.1 years old, S.D. $=11.3 ; \mathrm{p}<0.001)$. A higher percentage of psychiatry trainees were in the first year of the psychiatry residency $(36.1 \%, \mathrm{n}=119)$, with $28.8 \%(\mathrm{n}=95)$ in the second, $29.4 \%(\mathrm{n}=97)$ in the third, and the remaining $5.8 \%$ $(n=19)$ in the fourth year of residency. A similar proportion of men and women was found between groups and most of the 
psychiatry trainees were single $(93 \%, \mathrm{n}=307)$ with no children $(95.8 \%, \mathrm{n}=316)$ unlike psychiatrists (see Table 1).

\section{Professional Activities and Adversities}

Specific variables related to professional activities between groups are shown in Table 1. Clinical consultations in the government sector were more frequent, with more hours being spent on consultation by psychiatry trainees than by psychiatrists $(\mathrm{p}<0.001)$, while the latter reported more private clinical consultation and more hours spent on this activity $(\mathrm{p}<0.001)$. The maximum number of hours worked per day was higher in psychiatrists (13 h per day) than in psychiatry trainees $(12 \mathrm{~h}$, $\mathrm{p}<0.001)$.

Professional adversities from the start of the psychiatry residency were reported by most participants $(90.2 \%$, $n=618$ ): over $90 \%$ of psychiatrists and nearly $85 \%$ of psychiatry trainees $(\mathrm{p}<0.001)$. The most common adversity reported was having patients with severe suicidal ideation, attacks, being the attending physician of a patient who had committed suicide, and lawsuits. Most adversities were more frequently reported by psychiatrists. However, when assessing the type of attack and assailant, differences arose between groups: psychological and physical attacks were more common among psychiatry trainees as was being attacked by a patient, patient's relative, or professional colleague. Perceived discrimination was also higher among psychiatry trainees than among psychiatrists (Table 1).

\section{Physical and Mental Health Problems}

The presence of a physical health problem was more frequently reported in psychiatrists $(32.4 \%, \mathrm{n}=115)$ than in psychiatry trainees $(13.9 \%, \mathrm{n}=46: \mathrm{p}<0.001)$. Endocrine diseases (such as diabetes, hypothyroidism, and obesity) were the leading physical problems in both groups. In the group of psychiatrists, this was followed by cardiovascular diseases (such as hypertension and dysautonomia), whereas respiratory (asthma), gastrointestinal (gastritis and colitis), and musculoskeletal (such as hernias and lower back pain) diseases ranked second among psychiatry trainees (Table 2).

Over half the participants reported having a mental health problem $(62.8 \%$ of all participants, $n=430 ; 59.4 \%$ of psychiatry trainees, $n=196$ and $65.9 \%$ of psychiatrists, $n=234)$ during their professional careers (see Table 2). Major depression, anxiety disorders, and burnout were the most common mental

Table 1 Demographics, professional activities, and adversities between psychiatry trainees and psychiatrists

\begin{tabular}{|c|c|c|c|c|}
\hline & $\begin{array}{l}\text { Total } \\
\mathrm{n}=685\end{array}$ & $\begin{array}{l}\text { Psychiatry trainees } \\
\mathrm{n}=330\end{array}$ & $\begin{array}{l}\text { Psychiatrists } \\
\mathrm{n}=355\end{array}$ & Statistics \\
\hline \multicolumn{5}{|l|}{ Demographic features, n (\%) } \\
\hline Sex (women) & $355(51.8)$ & $175(53.0)$ & $180(50.7)$ & $\chi^{2}=0.3, p=0.54$ \\
\hline Age_-years (mean; S.D.) & $35.5 ; 10.7$ & $28.4 ; 2.1$ & $42.1 ; 11.3$ & $\mathrm{t}=22.3, \mathrm{p}<0.001 ;$ Cohen's $d=1.68$ \\
\hline Marital status (single) & $459(67.0)$ & $307(93.0)$ & $152(42.8)$ & $\chi^{2}=195.0, \mathrm{p}<0.001 ;$ Cramer's $\mathrm{V}=0.53$ \\
\hline Has children (yes) & $200(29.2)$ & $14(4.2)$ & $186(52.4)$ & $\chi^{2}=191.8, \mathrm{p}<0.001 ;$ Cramer's $\mathrm{V}=0.52$ \\
\hline \multicolumn{5}{|l|}{ Professional activities, $\mathrm{n}(\%)$} \\
\hline Consultation-government sector (yes) & $460(67.2)$ & $239(72.4)$ & $221(62.3)$ & $\chi^{2}=8.0, p=0.005 ;$ Cramer's $V=0.10$ \\
\hline Hours per week (mean; S.D.) & $28.0 ; 25.8$ & $34.4 ; 29.0$ & $22.1 ; 20.7$ & $\mathrm{t}=6.2, \mathrm{p}<0.001 ;$ Cohen's $d=0.48$ \\
\hline Consultation — private sector (yes) & $282(41.2)$ & $29(8.8)$ & $253(71.3)$ & $\chi^{2}=275.6, \mathrm{p}<0.001 ;$ Cramer's $\mathrm{V}=0.63$ \\
\hline Hours (mean; S.D.) & $8.0 ; 13.0$ & $0.9 ; 5.5$ & $14.7 ; 14.5$ & $\mathrm{t}=-16.5, \mathrm{p}<0.001 ;$ Cohen's $d=1.25$ \\
\hline Maximum working hours per day (mean; S.D.) & $12.8 ; 5.4$ & $12.3 ; 6.2$ & $13.2 ; 4.5$ & $\mathrm{t}=-2.1, \mathrm{p}=0.03 ;$ Cohen's $d=0.16$ \\
\hline \multicolumn{5}{|l|}{ Professional adversities, n (\%) } \\
\hline Patients w/ severe suicidal ideation (yes) & $603(88.0)$ & $278(84.2)$ & $325(91.5)$ & $\chi^{2}=8.6, p=0.003 ;$ Cramer's V=0.11 \\
\hline Patients who committed suicide (yes) & $243(35.5)$ & $86(26.1)$ & $157(44.2)$ & $\chi^{2}=24.6, \mathrm{p}<0.001 ;$ Cramer's $V=0.19$ \\
\hline Lawsuits (yes) & $120(17.5)$ & $60(18.2)$ & $60(16.9)$ & $\chi^{2}=0.19, p=0.66$ \\
\hline Attacks (yes) & $443(64.7)$ & $153(46.4)$ & $290(81.7)$ & $\chi^{2}=93.4, p<0.001 ;$ Cramer's $V=0.36$ \\
\hline Physical (yes) ${ }^{\mathrm{a}}$ & $220(49.7)$ & $89(58.2)$ & $131(45.2)$ & $\chi^{2}=6.7, \mathrm{p}=0.009 ;$ Cramer's V=0.12 \\
\hline Verbal (yes) ${ }^{\mathrm{a}}$ & $367(82.8)$ & $120(78.4)$ & $247(85.2)$ & $\chi^{2}=3.2, p=0.07$ \\
\hline Psychological (yes) ${ }^{\mathrm{a}}$ & $213(48.1)$ & $99(64.7)$ & $114(39.3)$ & $\chi^{2}=25.8, \mathrm{p}<0.001 ;$ Cramer's V $=0.24$ \\
\hline Assailant_patients (yes) ${ }^{\mathrm{a}}$ & $358(80.8)$ & $133(86.9)$ & $225(77.6)$ & $\chi^{2}=5.6, p=0.01 ;$ Cramer's V=0.11 \\
\hline Assailant_patient's relative (yes) ${ }^{\mathrm{a}}$ & $257(58.0)$ & $103(67.3)$ & $154(53.1)$ & $\chi^{2}=8.3, p=0.004 ;$ Cramer's V=0.13 \\
\hline Assailant — colleague (yes) ${ }^{\mathrm{a}}$ & $129(29.1)$ & $79(51.6)$ & $50(17.2)$ & $\chi^{2}=57.3, \mathrm{p}<0.001 ;$ Cramer's V=0.36 \\
\hline Perceived discrimination (mean; S.D.) & $28.2 ; 14.3$ & $35.6 ; 12.6$ & $21.3 ; 12.2$ & $\mathrm{t}=15.0, \mathrm{p}<0.001 ;$ Cohen's $d=1.15$ \\
\hline
\end{tabular}

${ }^{a} n=443$ from those who reported being attacked 
Table 2 Physical and mental health problems between psychiatry trainees and psychiatrists

\begin{tabular}{|c|c|c|c|c|}
\hline & $\begin{array}{l}\text { Total } \\
\mathrm{n}=685\end{array}$ & $\begin{array}{l}\text { Psychiatry trainees } \\
\mathrm{n}=330\end{array}$ & $\begin{array}{l}\text { Psychiatrists } \\
\mathrm{n}=355\end{array}$ & Statistics \\
\hline \multicolumn{5}{|l|}{ Physical health problems, n (\%) } \\
\hline Any physical health problem (yes) & $161(23.5)$ & $46(13.9)$ & $115(32.4)$ & $\chi^{2}=32.3, p<0.001 ;$ Cramer's V=0.21 \\
\hline Respiratory (yes) ${ }^{\mathrm{a}}$ & $31(19.3)$ & $8(17.4)$ & $23(20.0)$ & $\chi^{2}=0.1, p=0.70$ \\
\hline Endocrine $(y e s)^{\mathrm{a}}$ & $53(32.9)$ & $15(32.6)$ & $38(33.0)$ & $\chi^{2}=0.003, p=0.95$ \\
\hline Renal (yes) ${ }^{\mathrm{a}}$ & $3(1.9)$ & $2(4.3)$ & $1(0.9)$ & $\chi^{2}=2.1, p=0.14$ \\
\hline Cardiovascular (yes) ${ }^{\mathrm{a}}$ & $36(22.4)$ & $7(15.2)$ & $29(25.2)$ & $\chi^{2}=1.8, p=0.16$ \\
\hline Gastrointestinal (yes) $^{\mathrm{a}}$ & $26(16.1)$ & $8(17.4)$ & $18(15.7)$ & $\chi^{2}=0.07, p=0.78$ \\
\hline Neurological (yes) ${ }^{\mathrm{a}}$ & $8(5.0)$ & $3(6.5)$ & $5(4.3)$ & $\chi^{2}=0.3, p=0.56$ \\
\hline Musculoskeletal (yes) ${ }^{\mathrm{a}}$ & $27(16.8)$ & $8(17.4)$ & $19(16.5)$ & $\chi^{2}=0.01, p=0.89$ \\
\hline Other (yes) ${ }^{\mathrm{a}}$ & $20(12.4)$ & $6(13.0)$ & $14(12.2)$ & $\chi^{2}=0.02, p=0.88$ \\
\hline \multicolumn{5}{|l|}{ Mental health problems, n (\%) } \\
\hline Any mental health problem (yes) & $430(62.8)$ & $196(59.4)$ & $234(65.9)$ & $\chi^{2}=3.1, p=0.07$ \\
\hline Major depression (yes) ${ }^{\mathrm{b}}$ & $299(69.5)$ & $133(67.9)$ & $166(70.9)$ & $\chi^{2}=0.4, p=0.48$ \\
\hline Anxiety disorders (yes) ${ }^{\mathrm{b}}$ & $295(68.6)$ & $149(76.0)$ & $146(62.4)$ & $\chi^{2}=9.1, p=0.002 ;$ Cramer's V=0.14 \\
\hline Burnout (yes) ${ }^{\mathrm{b}}$ & $214(49.8)$ & $97(49.5)$ & $117(50.0)$ & $\chi^{2}=0.01, p=0.91$ \\
\hline Suicidal ideation (yes) ${ }^{\mathrm{b}}$ & $60(14.0)$ & $31(15.8)$ & $29(12.4)$ & $\chi^{2}=1.0, p=0.30$ \\
\hline Eating disorders (yes) ${ }^{\mathrm{b}}$ & $40(9.3)$ & $19(9.7)$ & $21(9.0)$ & $\chi^{2}=0.06, p=0.79$ \\
\hline Sleep disorders (yes) ${ }^{\mathrm{b}}$ & $187(43.5)$ & $90(45.9)$ & $97(41.5)$ & $\chi^{2}=0.8, p=0.35$ \\
\hline Trauma (yes) ${ }^{\mathrm{b}}$ & $32(7.4)$ & $13(6.6)$ & $19(8.1)$ & $\chi^{2}=0.3, p=0.55$ \\
\hline Other (yes) ${ }^{\mathrm{b}}$ & $38(8.8)$ & $18(9.2)$ & $20(8.5)$ & $\chi^{2}=0.05, p=0.81$ \\
\hline Received treatment (yes) ${ }^{\mathrm{b}}$ & $363(84.4)$ & $159(81.1)$ & $204(87.2)$ & $\chi^{2}=2.9, p=0.08$ \\
\hline Pharmacological (yes) ${ }^{\mathrm{c}}$ & $305(84.0)$ & 131 (82.4) & $174(85.3)$ & $\chi^{2}=0.56, p=0.45$ \\
\hline Self-administrated (yes) ${ }^{\mathrm{d}}$ & $126(41.3)$ & $52(39.7)$ & $74(41.5)$ & $\chi^{2}=0.24, p=0.61$ \\
\hline Psychotherapy (yes) ${ }^{b}$ & $294(81.0)$ & $121(76.1)$ & $173(84.8)$ & $\chi^{2}=4.3, p=0.03 ;$ Cramer's V=0.11 \\
\hline Perceived distress (mean; S.D.) & $48.4 ; 29.2$ & $53.2 ; 27.8$ & $43.8 ; 29.8$ & $\mathrm{t}=4.2, \mathrm{p}<0.001 ;$ Cohen's $d=0.32$ \\
\hline
\end{tabular}

${ }^{a} n=161$ from those who reported any physical health problem

${ }^{\mathrm{b}} \mathrm{n}=430$ from those who reported any mental health problem

${ }^{c} n=363$ from those who received treatment

${ }^{d} \mathrm{n}=305$ from those who received pharmacological treatment

health problems reported in both groups, with a higher proportion of anxiety disorders being found in psychiatry trainees (76.0\%, $\mathrm{n}=149$ vs. $62.4 \%, \mathrm{n}=146$ of those who reported mental health problems; $\mathrm{p}=0.002$ ). A similar percentage of participants received pharmacological and/or psychotherapeutic treatment for these problems. As shown in Table 2, pharmacological treatment was self-prescribed by over a third of the participants, with no differences being reported between psychiatry trainees and psychiatrists. Perceived distress was higher in psychiatry trainees.

\section{Self-care Behaviors}

Different patterns of self-care behaviors were found between psychiatry trainees and psychiatrists (see Table 3 ). The former reported more hypercaloric $(47.3 \%, \mathrm{n}=156$ vs. $38.9 \%, \mathrm{n}=138)$ or hypocaloric $(6.1 \%, \mathrm{n}=20$ vs. $2.8 \%, \mathrm{n}=10 ; \mathrm{p}=0.003)$ meals and less established eating schedules $(51.5 \% \mathrm{n}=170$ vs.
$40.0 \%, \mathrm{n}=142 ; \mathrm{p}=0.002)$ while the number of meals per day, three on average, was similar in both groups.

Over half the participants in both groups reported exercising regularly. More psychiatry trainees engaged in mixed (aerobic and anaerobic) exercise $(41.3 \%, n=71$ vs. $16.9, n=33)$ while psychiatrists reported more aerobic exercise $(76.4 \%$, $\mathrm{n}=149$, vs. $51.7 \%, \mathrm{n}=89 ; \mathrm{p}<0.001)$. Both groups exercised an average of three days per week with a mean duration of an hour and a half (Table 3).

Mean hours of sleep were 6.0 (S.D.=0.9) for psychiatry trainees and 6.4 (S.D.=1.0) for psychiatrists $(\mathrm{p}<0.001)$. More psychiatry trainees $(28.5 \%, \mathrm{n}=94)$ than psychiatrists $(16.6 \%$, $\mathrm{n}=59 ; \mathrm{p}<0.001)$ slept $5 \mathrm{~h}$ or less. A higher proportion of psychiatrists reported restful sleep $(63.4 \%, n=225)$ compared with psychiatry trainees $(54.8 \%, \mathrm{n}=181 ; \mathrm{p}=0.02)$. More psychiatry trainees reported having social activities outside the workplace $(57.9 \%, \mathrm{n}=290$ vs. $81.4 \%, \mathrm{n}=289, \mathrm{p}=0.01)$ (Table 3). 
Prediction of Self-reported Major Depression, Anxiety Disorders, and Burnout in Psychiatry Trainees and Psychiatrists

Demographic features, professional activities and adversities, physical health problems, perceived distress, and self-care behaviors affected psychiatry trainees and psychiatrists differently (see Table 4). As can be seen, two of the main risk factors for major depression, anxiety disorders, and burnout in both groups were having any physical health problem and not having restful sleep (except for anxiety disorders in psychiatrists), which can triple the risk of their occurrence. Women were at a higher risk of occurrence of major depression in both groups and a higher risk for anxiety disorders in psychiatry trainees. Clinical consultations in the government sector and the number of hours spent on this activity affected groups differently, with consultations in the government sector conferring a higher risk for the three assessed mental health problems in psychiatry trainees, while clinical consultations in the private sector increased the risk for anxiety disorders in psychiatrists and burnout in psychiatry trainees. Moreover, a greater number of working hours increased the risk for both groups for major depression and for anxiety disorders in psychiatrists. Higher perceived distress mainly affected psychiatry trainees but was also a risk factor for burnout in psychiatrists. Psychiatrists with high perceived discrimination were also at a higher risk for major depression. Eating patterns, particularly the consumption of hypo- or hypercaloric meals, were also a risk factor for depression, anxiety disorders, and burnout in psychiatrists.

\section{Discussion}

Our results indicate that a significant number of psychiatrists and psychiatry trainees have been affected by mental health problems during their academic training and professional lifespan. Anxiety disorders were reported by more than three quarters of the psychiatry trainees who answered the survey, while major depression was reported by over $65 \%$ of the sample and burnout by nearly $50 \%$. These results are consistent with previous studies [24-26].

Even though a high proportion of psychiatrists and psychiatry trainees had received treatment (pharmacological and/or psychotherapeutic) for the mental problem they experienced, approximately $40 \%$ under pharmacological treatment had self-prescribed this treatment. The rate of selfprescription among physicians in other studies varies dramatically from $7.6 \%$ in first-year specialists in training in one study [27] to over $60 \%$ in non-consultant hospital doctors [28]. As these authors note, many factors are likely to influence self-prescription, and show this to be closely related to the perceived need to work while sick, a phenomenon that is closely linked to working conditions, such as lack of

Table 3 Self-care behaviors between psychiatry trainees and psychiatrists

\begin{tabular}{|c|c|c|c|c|}
\hline & $\begin{array}{l}\text { Total } \\
\mathrm{n}=685\end{array}$ & $\begin{array}{l}\text { Psychiatry trainees } \\
\mathrm{n}=330\end{array}$ & $\begin{array}{l}\text { Psychiatrists } \\
\mathrm{n}=355\end{array}$ & Statistics \\
\hline \multicolumn{5}{|l|}{ Self-care behaviors, $\mathrm{n}(\%)$} \\
\hline \multicolumn{5}{|l|}{ Eating patterns } \\
\hline Number of meals per day (mean; S.D.) & $3.1 ; 0.8$ & $3.1 ; 0.7$ & $3.1 ; 0.9$ & $\mathrm{t}=-0.9, \mathrm{p}=0.33$ \\
\hline Type of diet & & & & $\chi^{2}=0.003, p=0.95$ \\
\hline Hypercaloric & $294(42.90$ & $156(47.3)$ & $138(38.9)$ & \\
\hline Hypocaloric & $30(4.4)$ & $20(6.1)$ & $10(2.8)$ & $\chi^{2}=11.3, p=0.003 ;$ Cramer's V=0.12 \\
\hline Calorie-balanced & $361(52.7)$ & $154(46.7)$ & $207(58.3)$ & \\
\hline Eating schedules (yes) & $373(54.5)$ & $160(48.5)$ & $213(60.0)$ & $\chi^{2}=9.1, p=0.002 ;$ Cramer's V=0.11 \\
\hline Exercise (yes) & $367(53.6)$ & $172(52.1)$ & $195(54.9)$ & $\chi^{2}=0.5, p=0.46$ \\
\hline \multicolumn{5}{|l|}{ Type of exercise ${ }^{\mathrm{a}}$} \\
\hline Aerobic & $238(64.9)$ & $89(51.7)$ & $149(76.4)$ & \\
\hline Anaerobic & $25(6.8)$ & $12(7.0)$ & $13(6.7)$ & $\chi^{2}=27.7, p<0.001 ;$ Cramer's V=0.27 \\
\hline Mixed & $104(28.3)$ & $71(41.3)$ & $33(16.9)$ & \\
\hline Days per week $^{\mathrm{a}}$ (mean; S.D.) & $3.5 ; 1.4$ & $3.3 ; 1.1$ & $3.7 ; 1.5$ & $\mathrm{t}=-2.5, \mathrm{p}=0.01$, Cohen's $d=0.30$ \\
\hline Time per session $^{\mathrm{a}}$ (h) (mean; S.D.) & $1.3 ; 0.6$ & $1.3 ; 0.6$ & $1.3 ; 0.6$ & $\mathrm{t}=0.7, \mathrm{p}=0.43$ \\
\hline \multicolumn{5}{|l|}{ Sleep } \\
\hline Number of hours (mean; S.D.) & $6.2 ; 1.0$ & $6.0 ; 0.9$ & $6.4 ; 1.0$ & $\mathrm{t}=-5.7, \mathrm{p}<0.001$, Cohen's $d=0.42$ \\
\hline Restful sleep (yes) & $406(59.3)$ & $181(54.8)$ & $225(63.4)$ & $\chi^{2}=5.1, p=0.02 ;$ Cramer's $V=0.08$ \\
\hline Social activities (yes) & $579(84.5)$ & $290(87.9)$ & $289(81.4)$ & $\chi^{2}=5.4, p=0.01 ;$ Cramer's V=0.08 \\
\hline
\end{tabular}

${ }^{a} n=367$ from those who exercise 
time to attend a consultation; difficulty finding a replacement if absent; and the accumulation of work if absent. Guille and Sen [27] point out that the reduction observed in their study in comparison with previous national studies in the USA may be influenced by the change in the number of working hours. Moreover, in the specific area of mental disease, stigma is another key factor in self-prescription [28]. Self-stigma and fear of being stigmatized by colleagues could explain this phenomenon. They prefer to self-prescribe than be exposed to discrimination, isolation, lack of compassion and understanding, or being deemed incompetent, and are concerned about jeopardizing their status or having their privacy and autonomy violated [29]. Despite ethical and practical concerns, self-prescription is particularly worrisome in our sample of psychiatry trainees since most of them $(65 \%)$ were only just beginning their specialization, meaning that their diagnosis and treatment choices are questionable, with a higher probability of adverse events related to treatment and a higher probability of treatment inefficacy. Psychiatrists' treatment selection may be based on the profile of the side effects of the medication of choice and be inconsistent with symptom severity. Furthermore, our study did not include the issue of which medications were used to treat this condition, leaving open the possibility of treatment based solely on symptomatic relief (i.e., with benzodiazepines or stimulants rather than SSRIs). In addition, medical knowledge makes doctors prone to oscillating between panic and denial when experiencing symptoms [30], leading to a clear necessity of encouraging treatment and discouraging self-prescription, perhaps through the implementation of mandatory routine medical consultations alongside restrictions for self-prescription of sedatives and stimulants.

In keeping with gender-related vulnerability to mental illness [31], women are at a higher risk for major depression and anxiety disorders [13, 32], particularly psychiatry trainees, as age appears to be a protective factor for anxiety disorders in psychiatrists.

Professional activities and adversities impact differently on psychiatry trainees and psychiatrists. Having clinical consultations in the government sector was an important risk factor for major depression and burnout in psychiatry trainees. Training is stressful and the experience of high levels of responsibility combined with the lack of professional experience (especially since most trainees were in their first and second years of training) [33] may increase distress and lead to increased risks of mental health problems, as borne out by our results showing the impact of perceived distress on this group. A certain amount of discomfort is to be expected as part of all learning experiences. However, the increased risk of mental health problems may indicate that particular attention should be paid to how students are coping with the training experience as a psychiatric specialist and determining whether additional support is needed.
Having patients with severe suicidal ideation was the most common professional adversity reported by psychiatrists and psychiatry trainees and conferred a higher risk for having an anxiety disorder in the latter. Dealing with patients who express suicidal intention seems to be the greatest difficulty psychiatrists can face in their profession $[34,35]$. It is a particularly difficult stressor to cope with [36], with reports of severe stress levels (comparable to the loss of a parent) in treating therapists, a substantial proportion of whom develop posttraumatic symptoms when a patient under their care commits suicide [37]. A sense of competence and confirmation of professional skills (when faced with this adversity) can only come from experience and training [35]. Accordingly, close supervision by a more experienced or attending psychiatrist working with established institutional protocols for providing additional resources to trainees to manage suicidal patients and reduce anxiety is required, together with help for them to deal with guilt and genuinely consider whether mistakes were made [35]. The high discrimination reported by trainees (mean score $=35.6$ vs . 21.3 of psychiatrists) and attacks by colleagues (51.6\%) evidence the need for assessing how this supervision takes place, as it does not appear to be providing the constructive feedback one would expect in teaching facilities.

Psychiatrists show better measures of self-care than psychiatrists in training. Doctors often suggest a balanced diet, moderate exercise, and $8 \mathrm{~h}$ of sleep a night to their patients yet neglect to follow their own advice.

Physicians also sleep less than they might need to on a regular basis [38]. Contrary to other studies, in our group of psychiatry trainees, sleeping less than $5 \mathrm{~h}$ was not a risk factor for anxiety and burnout symptoms. This phenomenon is probably the result of medical culture, where the dominant idea in the medical profession is that physicians are never sick and, if they are, they must work silently through their illness and put patient care above everything else. There is a definite need to continue to promote self-care in the medical profession and to debunk the myth of the infallible physician.

The limitations of our study come from three main sources: self-reporting rather than active evaluation and detection of these mental health problems, lack of randomization of the sample, and its retrospective nature.

Self-reporting of these problems should be taken with caution as it is likely to include other problems that may not meet clearly defined diagnostic criteria. For example, self-reported major depression may include depression or dysthymia [39], while burnout may be indistinguishable from depressive or anxious symptoms [40]. Coupled with the retrospective design of the study, this makes it impossible to determine how accurate these diagnoses are or whether they are currently present. Moreover, in the self-diagnosis process, qualitative studies have shown that physicians tend to under- or over-react to their symptoms, switching from diagnosing diseases with the worst prognosis to ruling out a disorder altogether [30]. Medical 
Table 4 Factors associated with self-reported major depression, anxiety disorders, and burnout in psychiatry trainees and psychiatrists

\begin{tabular}{|c|c|c|c|c|c|c|}
\hline & \multicolumn{3}{|c|}{ Psychiatry trainees } & \multicolumn{3}{|c|}{ Psychiatrists } \\
\hline & OR & 95\% C.I. & & OR & $95 \%$ C.I & \\
\hline \multicolumn{7}{|l|}{ Major depression } \\
\hline Sex (women) & $3.08 * * *$ & 1.78 & 5.32 & $2.07 * *$ & 1.27 & 3.37 \\
\hline Marital status (single) & $0.37 *$ & 0.13 & 1.02 & & & \\
\hline Consultation—government sector (yes) & $6.54 * * *$ & 2.59 & 16.50 & & & \\
\hline Hours of consultation — government sector & $0.97 * *$ & 0.96 & 0.99 & & & \\
\hline Maximum working hours per day & $1.06^{* *}$ & 1.01 & 1.11 & $1.07 * *$ & 1.02 & 1.14 \\
\hline Attacks (yes) & & & & $1.93 *$ & 0.99 & 3.76 \\
\hline Perceived discrimination & & & & $1.02 * *$ & 1.005 & 1.04 \\
\hline Any physical health problem (yes) & $2.67 * *$ & 1.28 & 5.55 & $1.59 *$ & 0.96 & 2.63 \\
\hline Perceived distress & $1.02 * * *$ & 1.01 & 1.03 & & & \\
\hline Meals per day (less than 3 ) & & & & $0.51 * *$ & 0.27 & 0.97 \\
\hline Type of diet (hyper- or hypocaloric) & & & & $1.83 * *$ & 1.06 & 3.17 \\
\hline Eating schedules (no) & & & & $1.98 * *$ & 1.15 & 3.39 \\
\hline Restful sleep (no) & $2.68 * * *$ & 1.55 & 4.62 & $1.88 * *$ & 1.14 & 3.10 \\
\hline Social activities (no) & $2.46^{* *}$ & 1.08 & 5.56 & & & \\
\hline Constant & 0.03 & & & 0.03 & & \\
\hline \multicolumn{7}{|c|}{$\begin{array}{l}\text { Psychiatry trainees: Nagelkerke } \mathrm{R}^{2}=0.337 ; \text { Hosmer and Lemeshow test } \mathrm{p}=0.169 \\
\text { Psychiatrists: Nagelkerke } \mathrm{R}^{2}=0.238 ; \text { Hosmer and Lemeshow test } \mathrm{p}=0.786\end{array}$} \\
\hline \multicolumn{7}{|l|}{ Anxiety disorders } \\
\hline Sex (women) & $2.30 * *$ & 1.38 & 3.81 & 1.44 & 0.89 & 2.34 \\
\hline Current age & & & & $0.95 * * *$ & 0.92 & 0.97 \\
\hline Marital status (single) & 0.48 & 0.17 & 1.32 & & & \\
\hline Hours of consultation - government sector & $1.00 *$ & 1.000 & 1.01 & & & \\
\hline Consultation—private sector (yes) & & & & $2.62 * *$ & 1.33 & 5.18 \\
\hline Hours of consultation-private sector & & & & 0.98 & 0.96 & 1.00 \\
\hline Maximum working hours per day & & & & $1.04 *$ & 0.99 & 1.09 \\
\hline Patients with severe suicidal ideation (yes) & $2.18^{* *}$ & 1.007 & 4.75 & & & \\
\hline Attacks (yes) & $1.65^{*}$ & 0.92 & 2.97 & & & \\
\hline Any physical health problem (yes) & $3.04 * *$ & 1.45 & 6.37 & $2.64 * * *$ & 1.59 & 4.40 \\
\hline Perceived distress & $1.02 * * *$ & 1.01 & 1.03 & & & \\
\hline Type of diet (hyper- or hypocaloric) & & & & $1.67 * *$ & 1.03 & 2.71 \\
\hline Number of hours of sleep (less than $5 \mathrm{~h}$ ) & $0.50 * *$ & 0.27 & 0.93 & & & \\
\hline Restful sleep (no) & $2.69 * * *$ & 1.56 & 4.65 & & & \\
\hline Social activities (no) & & & & $1.73 *$ & 0.95 & 3.14 \\
\hline Constant & 0.06 & & & 0.81 & & \\
\hline \multicolumn{7}{|c|}{$\begin{array}{l}\text { Psychiatry trainees: Nagelkerke } R^{2}=0.300 \text {; Hosmer and Lemeshow test } p=0.574 \\
\text { Psychiatrists: Nagelkerke } R^{2}=0.206 \text {; Hosmer and Lemeshow test } p=0.159\end{array}$} \\
\hline \multicolumn{7}{|l|}{ Burnout } \\
\hline Consultation - government sector (yes) & $2.69 * *$ & 1.39 & 5.23 & & & \\
\hline Hours of private clinical consultation & $1.05^{*}$ & 0.99 & 1.11 & & & \\
\hline Patients with severe suicidal ideation (yes) & & & & $0.45 *$ & 0.19 & 1.08 \\
\hline Perceived discrimination & & & & $1.02 *$ & 0.99 & 1.04 \\
\hline Any physical health problem (yes) & $4.69 * * *$ & 2.28 & 9.67 & $2.77 * * *$ & 1.61 & 4.77 \\
\hline Perceived distress & $1.02 * * *$ & 1.01 & 1.03 & $1.01 * *$ & 1.004 & 1.02 \\
\hline Type of diet (hyper- or hypocaloric) & & & & $1.69 * *$ & 1.001 & 2.86 \\
\hline Eating schedules (no) & $0.58^{*}$ & 0.33 & 1.04 & & & \\
\hline Number of hours of sleep (less than $5 \mathrm{~h}$ ) & $0.51 * *$ & 0.27 & 0.97 & & & \\
\hline
\end{tabular}


Table 4 (continued)

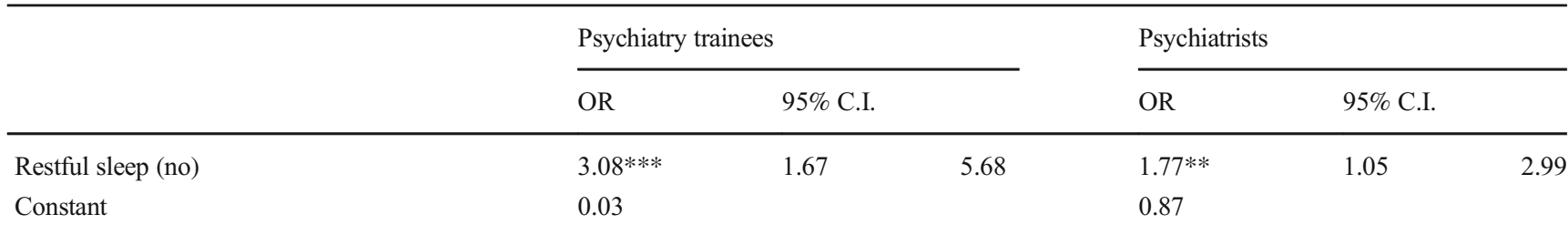

Psychiatry trainees: Nagelkerke $\mathrm{R}^{2}=0.259$; Hosmer and Lemeshow test $\mathrm{p}=0.720$

Psychiatrists: Nagelkerke $R^{2}=0.284$; Hosmer and Lemeshow test $\mathrm{p}=0.397$

${ }^{*} p \leq 0.1, * * p \leq 0.05, * * * p \leq 0.001$

culture may encourage both under-reporting and underrecognition of illness and burnout. Although the anonymous nature of the survey may encourage more accurate selfreporting, the extent of under-recognition is impossible to determine given the design of this study. Nevertheless, since the study subjects are experts in the field of mental health, these self-reports give cause for concern and should be carefully considered.

Lack of randomization of the sample could lead to a bias in the interpretation of our results. Even though most of the psychiatrists and psychiatry trainees come from the three main cities where these professionals are concentrated in the country (Mexico City 66.6\%; Jalisco 10.5\%; and Nuevo León 2.8\%) [19], we cannot rule out the possibility that those who answered the survey were those with clearly identified mental health problems. Also, we had no participants from three states in this survey (Hidalgo, Guerrero, and Tlaxcala). Compared to the remaining states, these have very few psychiatrists [19] and rather than cultural or economic reasons, we think possible participants did not receive the link for the survey or did not want to participate. Accordingly, our data should be taken with caution and cannot be generalized to the universe of psychiatrists and psychiatry trainees in our country.

The retrospective nature of the study limits the study of certain variables as risk factors, such as lack of restful sleep, which could be both a risk factor or a current manifestation of depression, anxiety, or burnout. Future studies should evaluate current symptoms to address this concern.

The medical world must evolve towards the recognition that physicians are only human to cultivate integrity, self-reflection, and the ability to admit weaknesses and mistakes while striving for continuous improvement and learning. In addition, physicians with healthy habits are more likely to provide appropriate preventive care and counseling to their patients.

Encouraging psychiatrists to take better care of themselves is just one piece of the puzzle, which must be accompanied by tangible organizational avenues to do so and the creation of a culture that truly promotes self-care. We must continue to engage in honest dialogue to raise awareness, while enacting policies that regulate working hours, workload, and work environment, and promote proper work-life balance (for example, daycare support), and ongoing screening of personnel in training followed by a flowchart for both prevention and care. In turn, we need to take time to restructure the hierarchical medical culture permeated by the normalization of violent behaviors disguised as necessary strategies for teachinglearning medicine. Redefining the idea of sacrifice and the overevaluation of academic and professional achievements over self-care is imperative; in other words, shifting the paradigm of forming the "medical character" with an irreversible loss of physical and mental health is mandatory. We believe these goals might be attained through medical wellness programs, started from medical school but maintained in clinical settings, focusing on de-stigmatizing physical and mental illness in doctors, promoting help-seeking behaviors, and addressing the risks of self-diagnosis and self-medication. The steps we take today will shape the future of medicine and psychiatry for years to come.

Acknowledgements The authors would like to thank "Divulgación científica de trastornos psiquiátricos" S.C. for providing the platform for surveys.

\section{Declarations}

Ethical Considerations All procedures comply with the ethical standards of the relevant national and institutional committees on human experimentation and were approved by the Ethics and Research Committees

Disclosures On behalf of all authors, the corresponding author states that there is no conflict of interest.

\section{References}

1. Wallace JE, Lemaire JB, Ghali WA. Physician wellness: a missing quality indicator. Lancet. 2009;374(9702):1714-21.

2. McFarland DC, Hlubocky F, Riba M. Update on addressing mental health and burnout in physicians: what is the role for psychiatry? Curr Psychiatry Rep. 2019;21(11):108.

3. Hawton K, Clements A, Sakarovitch C, Simkin S, Deeks JJ. Suicide in doctors: a study of risk according to gender, seniority and specialty in medical practitioners in England and Wales, 19791995. J Epidemiol Community Health. 2001;55(5):296-300. 
4. Umene-Nakano W, Kato TA, Kikuchi S, Tateno M, Fujisawa D, Hoshuyama T, et al. Nationwide survey of work environment, work-life balance and burnout among psychiatrists in Japan. PLoS One. 2013;8(2):1-16.

5. Olkinuora M, Asp S, Juntunen J, Kauttu K, Strid L, Äärimaa M. Stress symptoms, burnout and suicidal thoughts in Finnish physicians. Soc Psychiatry Psychiatr Epidemiol. 1990;25(2):81-6.

6. Martínez-López JNI, Robles R, Fresán A, de Montis IA, Kismödi E, Medina-Mora ME, et al. Legal and policy implications in Mexico of changes in ICD-11 paraphilic disorders. J Sex Med. 2019;16(10):1623-37.

7. Heponiemi T, Aalto AM, Puttonen S, Vänskä J, Elovainio M. Work-related stress, job resources, and well-being among psychiatrists and other medical specialists in Finland. Psychiatr Serv. 2014;65(6):796-801.

8. Murdoch JM, Eagles JM. "Stress-busting" groups for consultant psychiatrists. Psychiatr Bull. 2007;31(4):128-31.

9. Kumar S. Burnout in psychiatrists. World Psychiatry. 2007;6(3): 186-9.

10. Boscarino JA, Adams RE, Figley CR. Secondary trauma issues for psychiatrists. Psychiatr Times. 2010;27(11):24-6.

11. Bowers L, Simpson A, Eyres S, Nijman H, Hall C, Grange A, et al. Serious untoward incidents and their aftermath in acute inpatient psychiatry: the Tompkins Acute Ward Study. Int J Ment Health Nurs. 2006;15(4):226-34.

12. Ruskin R, Sakinofsky I, Bagby RM, Dickens S, Sousa G. Impact of patient suicide on psychiatrists and psychiatric trainees. Acad Psychiatry. 2004;28(2):104-10.

13. Jovanović N, Beezhold J, Tateno M, Barrett E, Vlachos I, Fiorillo $\mathrm{A}$, et al. Depression and suicidality among psychiatric residents results from a multi-country study. J Affect Disord. 2019;249: 192-8.

14. Mata DA, Ramos MA, Bansal N, Khan R, Guille C, Di Angelantonio E, et al. Prevalence of depression and depressive symptoms among resident physicians: a systematic review and meta-analysis. JAMA. 2015;314(22):2373-83.

15. Coverdale J, Balon R, Beresin EV, Brenner AM, Louie AK, Guerrero APS, et al. What are some stressful adversities in psychiatry residency training, and how should they be managed professionally? Acad Psychiatry. 2019;43(2):145-50.

16. Jovanović N, Podlesek A, Volpe U, Barrett E, Ferrari S, Rojnic Kuzman M, et al. Burnout syndrome among psychiatric trainees in 22 countries: risk increased by long working hours, lack of supervision, and psychiatry not being first career choice. Eur Psychiatry. 2016;32:34-41.

17. Monteiro G, Passos I, Baeza F, Hauck S. Burnout in psychiatry residents: the role of relations with peers, preceptors, and the institution. Braz J Psychiatry. 2020;42:226-7.

18. Córdoba RN, Cano JF, Alzate M, Olarte AF, Salazar I, Cendales R. El médico psiquiatra latinoamericano: su perfil y su grado de satisfacción con la especialidad. Actas Esp Psiquiatr. 2009;37(1): 9-16.

19. Heinze G, Bernard-Fuentes N, Carmona-Huerta J, Chapa G. del C, Guízar-Sánchez DP. Physicians specializing in psychiatry of Mexico: an update 2018. Salud Ment. 2019;42(1):13-23.

20. Terrones-Rodríguez JF, Cisneros-Pérez V, Arreola-Rocha JJ. Síndrome de burnout en médicos residentes del Hospital General de Durango, México. Rev Med Inst Mex Seguro Soc. 2016;54(2): $242-8$.

21. Toral-Villanueva R, Aguilar-Madrid G, Juárez-Pérez CA. Burnout and patient care in junior doctors in Mexico City. Occup Med. 2009;59(1):8-13.

22. Flores Reynoso S, Medina Dávalos R, Robles GR. Estudio de traducción al español y evaluación psicométrica de una escala para medir el estigma internalizado en pacientes con trastornos mentales graves. Salud Ment. 2011;34(4):333-9.

23. King M, Dinos S, Shaw J, Watson R, Stevens S, Passetti F, et al. The Stigma Scale: development of a standardised measure of the stigma of mental illness. Br J Psychiatry. 2007;190:248-54.

24. Hardy P, Devouge I, Pelissolo A, Corruble E. Psychiatry: a discipline at specific risk of mental health issues? J Affect Disord. 2019;254:120-1.

25. Fond G, Bourbon A, Micoulaud-Franchi JA, Auquier P, Boyer L, Lançon C. Psychiatry: a discipline at specific risk of mental health issues and addictive behavior? Results from the national BOURBON study. J Affect Disord. 2018;238:534-8.

26. Rotstein S, Hudaib AR, Facey A, Kulkarni J. Psychiatrist burnout: a meta-analysis of Maslach Burnout Inventory means. Australas Psychiatry. 2019;27(3):249-54.

27. Guille C, Sen S. Prescription drug use and self-prescription among training physicians. Arch Intern Med. 2012;172(4):371-372.

28. Feeney S, O'Brien K, O'Keeffe N, Iomaire ANC, Kelly ME, McCormack J, et al. Practise what you preach: health behaviours and stress among non-consultant hospital doctors. Clin Med. 2016;16(1):12-8.

29. White A, Shiralkar P, Hassan T, Galbraith N, Callaghan R. Barriers to mental healthcare for psychiatrists. Psychiatr Bull. 2006;30(10): $382-4$.

30. Thompson WT, Cupples ME, Sibbett CH, Skan DI, Bradley T. Challenge of culture, conscience, and contract to general practitioners' care of their own health: qualitative study. Br Med J. 2001;323(7315):728-31.

31. Seedat S, Scott KM, Angermeyer MC, Berglund P, Bromet EJ, Brugha TS, et al. Cross-national associations between gender and mental disorders in the World Health Organization World Mental Health Surveys. Arch Gen Psychiatry. 2009;66(7):785-95.

32. Guille C, Frank E, Zhao Z, Kalmbach DA, Nietert PJ, Mata DA, et al. Work-family conflict and the sex difference in depression among training physicians. JAMA Intern Med. 2017;177(12): 1766-72.

33. Firth-Cozens J, Payne R. In: Firth-Cozens J, Payne R, editors. Stress in health professionals: psychological and organizational causes and interventions. 2nd ed. London: Wiley; 2000.

34. Hendin H, Lipschitz A, Maltsberger JT, Haas AP, Wynecoop S. Therapists' reactions to patients' suicides. Am J Psychiatry. 2000;157(12):2022-7.

35. Scupham S, Goss SP. Working with suicidal clients: psychotherapists and allied professionals speak about their experiences. Couns Psychother Res. 2020;20(3):525-34.

36. Fothergill A, Edwards D, Burnard P. Stress, burnout, coping and stress management in psychiatrists: findings from a systematic review. Int J Soc Psychiatry. 2004;50(1):54-65.

37. Chemtob CM, Hamada RS, Bauer G, Kinney B, Torigoe RY. Patients' suicides: frequency and impact on psychiatrists. Am J Psychiatry. 1988;145(2):224-8.

38. Coleman JJ, Robinson CK, Zarzaur BL, Timsina L, Rozycki GS, Feliciano DV. To sleep, perchance to dream: acute and chronic sleep deprivation in acute care surgeons. J Am Coll Surg. 2019;229(2):166-74.

39. Korkeila JA, Töyry S, Räsänen K, Kumpulainen K, Toivola JM, Kalimo R. Burnout and self-perceived health among Finnish psychiatrists and child psychiatrists: a national survey. Scand J Public Health. 2003;31(2):85-91.

40. Schonfeld IS, Bianchi R. Burnout and depression: two entities or one? J Clin Psychol. 2016;72(1):22-37.

Publisher's Note Springer Nature remains neutral with regard to jurisdictional claims in published maps and institutional affiliations. 